Sharif University of Technology
Scientia Iranica
SCIENTIA
I RAN ICA
http://scientiairanica.sharif.edu

\title{
Space headway calculation and analysis at turn movement trajectories using hybrid model
}

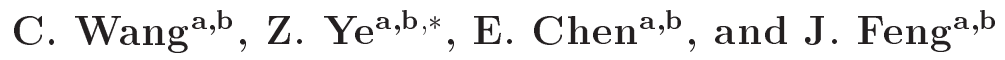 \\ a. Jiangsu Key Laboratory of Urban ITS, Southeast University, Nanjing, China. \\ b. Jiangsu Province Collaborative Innovation Center of Modern Urban Traffic Technologies, Southeast University, Nanjing, China. \\ Received 29 July 2016; received in revised form 17 October 2018; accepted 3 March 2019
}

\author{
KEYWORDS \\ Space headway; \\ Spline curve; \\ Numerical integration; \\ Car-following; \\ Turn movement \\ trajectories.
}

\begin{abstract}
Space headway calculation and analysis play an important role in identifying surrounding obstacles and understanding traffic scenes. However, the performance of existing methods is limited by the complexity of computer processing. In addition, it is quite difficult to obtain space headway at turn movement trajectories, mainly owing to the limitation of rectilinear propagation. Therefore, a hybrid model based on spline curve and numerical integration was proposed to estimate the distance of the front vehicle and vehicle trajectory in this study. The space headway at turn movement trajectories was analogous to the track of a vehicle, which could be fitted by a quadratic spline curve. Newton-Cotes numerical integration was employed to calculate distance due to its meshing flexibility and ease of implementation. Data collected from Lankershim Boulevard in the city of Los Angeles, California (USA) were used to evaluate performance of the hybrid model. Compared with another algorithm based on computer vision and trilinear method, the results showed that the proposed model worked successfully and outperformed the competing method in terms of accuracy and reliability. Finally, the proposed method was applied to investigate the effects of vehicle speed, relative speed of vehicles, and time period on the spacing of vehicles during car-following.

(C) 2020 Sharif University of Technology. All rights reserved.
\end{abstract}

\section{Introduction}

Space headway is the distance between adjacent vehicles front-to-back traveling in the same direction. A growing number of research scholars focus on the study of calculating space headway, since it plays an important role in traffic safety [1-3]. Safe driving research mainly consists of front-vehicle detection [4], lateral collision avoidance [5], longitudinal collision avoidance $[6,7]$, driver state monitoring, and lane estimation for autonomous driving [8]. Accurate estimation of

\footnotetext{
*. Corresponding author. Tel.: +862583790583; Fax: + 862583794102

E-mail address: yezhirui@seu.edu.cn (Z.Ye)
}

vehicle path and lane position is of importance to autonomous driving and it can be simplified as a curve estimation problem, where the measured data provide partial and noisy observations of spatial curves forming lane boundaries. The challenges lie in detecting vehicle path and lane position from local sensor data and updating them as new observations are made [9]. In addition, most traffic accidents result from distraction, namely inattention to surrounding cars. To protect drivers, it is necessary to monitor road conditions and provide lateral and longitudinal control to avoid collisions [10]. Consequently, as the premise of safe driving, calculating and analyzing space headway is absolutely essential and makes it possible to identify surrounding obstacles and to understand the traffic scene in the car-following behavior [11-13]. In this study, a hybrid model based on spline curve and 
numerical integration is proposed to solve the space headway calculation problem and it can be applied to estimate the distance to the front vehicle and vehicle path estimation.

In early research on space headway, several categories of methodologies have been developed for measurement and calculation, such as laser ranging $[14,15]$, ultrasonic ranging [16], and radar ranging [17]. Compared to the above methods, computer vision technology is capable of acquiring more accurate and reliable results [18]. It provides an alternative approach with the advantages of automated processing and a non-destructive procedure to address the question. Stauffer and Grimson [19] and Kilger 20] developed a background subtraction method to identify the front vehicle. However, when illumination conditions were similar, the selection between various algorithms was difficult. Zhao et al. [21] proposed a segmentation approach based on inner-frame difference and a modified computer vision model. Their methods could shorten computation time and avoid segmenting the entire image. An optical flow and clustering algorithm was presented for spatio-temporal image segmentation. It was tested on images with mobile vehicles and backgrounds, which solved the dilemma of similar background conditions. However, the investigation of other features and clustering techniques should be discussed [22]. Region tracking and motion-based segmentation are used to estimate the trajectories of vehicles, while issues related to large vehicles and large shadows require further research [23]. In addition, many methods [24-27] have also been applied to measuring and calculating the distance of space headway. For instance, an algorithm based on a trilinear method [27] was proposed to measure the preceding vehicle distance through several extrinsic parameters and perspective projection geometry. However, in these methods, many parameters of the camera need to be available such as mounting height, effective focal length, and tilt angle [28]. This method assumes that the ground is flat and that the vehicle coordinate system is in accordance with the world coordinate system and so forth; therefore, some errors will be produced.

As demonstrated by the previous research described above, a variety of methods have been used to estimate the distance to the front vehicle in straight segments of roadway. Nevertheless, it is computationally complex and quite difficult to obtain space headway at turn movement trajectories, mainly owing to the limitation of rectilinear propagation and the complexity of computer processing. In order to simplify the operation process and achieve more reliable and accurate results, a hybrid model based on a quadratic spline curve and Newton-Cotes numerical integration is proposed. The method is flexible for modeling object shapes and suitable for solving the space headway problem at turn movement trajectories. In addition, the hybrid model offers reasonable compromise between flexibility and speed of computation. Compared to computer vision technology, the method is more stable and requires less computation and storage space.

The space headway at turn movements is analogous to the track of a vehicle, which is difficult to fit by a single polynomial [29]. As a widely adopted standard in many research efforts [30-33], a spline curve is frequently used to solve the curve-fitting and data-fitting problem. It should be noted that complicated computations may be generated when selecting a cubical or higher spline curve, a B-spline curve, and a rational spline curve. For this reason, a quadratic spline curve is a good choice. Then, Newton-Cotes numerical integration is employed to calculate the distance. Although Gaussian method [34] has a slightly more favorable performance than the Newton-Cotes method in some fields such as plant morphology, it is unsuitable for vehicle trajectories, which have simpler linetype compared with the plant morphology. The Newton-Cotes integration is appropriate to address this study due to its meshing flexibility and ease of implementation. In addition, vehicle path estimation can be formulated as the above quadratic spline curve by obtaining a set of position coordinates (characteristic points). These coordinates are then fitted with piecewise-continuous, parametric polynomial functions by using interpolation methods. The polynomial sections are fitted so that all the characteristic points are connected. Consequently, the hybrid model is efficient for space headway calculation and vehicle path estimation, especially at turn movement trajectories.

The remaining sections of the paper are organized as follows. Section 2 presents the method based on a quadratic spline curve and Newton-Cotes numerical integration. In Section 3, vehicle trajectory data collected from urban roads are used to evaluate performance of the proposed method. Section 4 discusses results and future research.

\section{Methodology}

\subsection{Quadratic spline curve}

In general terms, the objective of the spline curvefitting problem is to generate a fitted curve to approximate a target curve, whose form is controlled by a series of data points, namely characteristic points. The number of characteristic points can be determined by the object contour and shape of the curve. In research on space headway, the target curve represents the trajectories of turning vehicles at intersections and the data points are the vehicle coordinates, which are defined on a 2D plane. Assuming that $P(t)=(\phi(t), \psi(t))$ denotes the arbitrary point coordinate, it has a first- 
order derived function of $P^{\prime}(t)=\left(\phi^{\prime}(t), \psi^{\prime}(t)\right)$. The quadratic spline curve is expressed as follows:

$$
P(t)=A_{1}+A_{2} t+A_{3} t^{2} \quad(0 \leq t \leq 1),
$$

where $t$ is a parametric variable. For a given parametric curve trajectory, it is unnecessary to investigate the entire value range of $t$. The researchers are interested in a certain value interval in most cases. Thus, normalized transformation is used so that $t$ falls between 0 and 1 . $A_{1}, A_{2}$, and $A_{3}$ denote three-point coordinates, which are defined on a $2 \mathrm{D}$ plane. Let:

$$
\begin{aligned}
& t=0: P(0)=A_{1}=P_{1}=\left(\phi_{1}, \psi_{1}\right) \\
& t=0.5: P(0.5)=A_{1}+0.5 A_{2}+0.25 A_{3}=P_{2}=\left(\phi_{2}, \psi_{2}\right), \\
& t=1: P(1)=A_{1}+A_{2}+A_{3}=P_{3}=\left(\phi_{3}, \psi_{3}\right) .
\end{aligned}
$$

By solving the simultaneous equations, the quadratic spline curve can be represented as follows:

$$
\begin{aligned}
P(t) & =P_{1}+\left(4 P_{2}-P_{3}-3 P_{1}\right) t+\left(2 P_{1}+2 P_{3}-4 P_{2}\right) t^{2} \\
& =\left(2 t^{2}-3 t+1\right) P_{1}+\left(4 t-4 t^{2}\right) P_{2}+\left(2 t^{2}-t\right) P_{3} .
\end{aligned}
$$

In order to intuitively describe the arbitrary point coordinate $P(t)=(\phi(t), \psi(t))$, the authors obtain the matrix that characterizes this spline curve by rewriting Eq. (3) as the following matrix product:

$$
\begin{aligned}
{\left[\begin{array}{ll}
\phi(t) & \psi(t)
\end{array}\right] } & =\left[\begin{array}{lll}
t^{2} & t & 1
\end{array}\right]\left[\begin{array}{ccc}
2 & -4 & 2 \\
-3 & 4 & -1 \\
1 & 0 & 0
\end{array}\right] \\
& {\left[\begin{array}{cc}
\phi_{1} & \psi_{1} \\
\phi_{2} & \psi_{2} \\
\phi_{3} & \psi_{3}
\end{array}\right] . }
\end{aligned}
$$

This equation transforms the geometric constraint values to polynomial coefficients and provides a characterization for the spline curve. It contains characteristic point coordinate values and other geometric constraints that have been specified [35].

Given a series of discrete data points $P_{i}(i=$ $1,2, \ldots, n)$, every 3 adjacent points will form a certain curve trajectory. Therefore, the target curve is composed of $n-2$ segments if there are $n$ discrete data points (i.e., characteristic points). In the $n-$ 2 segments, the $i$ th segment $Q_{i}\left(t_{i}\right)$ is defined by 3 adjacent points $P_{i}, P_{i+1}, P_{i+2}$. Thus, the quadratic spline curve $Q_{i}\left(t_{i}\right)$ is:

$$
\begin{aligned}
Q_{i}\left(t_{i}\right)= & \left(2 t_{i}^{2}-3 t_{i}+1\right) P_{i}+\left(4 t_{i}-4 t_{i}^{2}\right) P_{i+1} \\
& +\left(2 t_{i}^{2}-t_{i}\right) P_{i+2} \quad\left(0 \leq t_{i} \leq 1\right) .
\end{aligned}
$$

In the same way, the $i+1$ th segment $Q_{i+1}\left(t_{i+1}\right)$ is defined by $P_{i+1}, P_{i+2}, P_{i+3}$ :

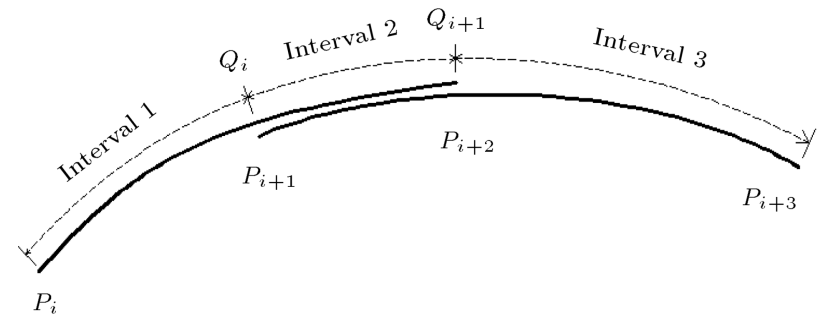

Figure 1. Curve trajectories $Q_{i}\left(t_{i}\right)$ and $Q_{i+1}\left(t_{i+1}\right)$.

$$
\begin{aligned}
& Q_{i+1}\left(t_{i+1}\right)=\left(2 t_{i+1}^{2}-3 t_{i+1}+1\right) P_{i+1} \\
& \quad+\left(4 t_{i+1}-4 t_{i+1}^{2}\right) P_{i+2}+\left(2 t_{i+1}^{2}-t_{i+1}\right) P_{i+3} \\
& \quad\left(0 \leq t_{i+1} \leq 1\right) .
\end{aligned}
$$

As mentioned above, the curve trajectories $Q_{i}\left(t_{i}\right)$ and $Q_{i+1}\left(t_{i+1}\right)$ produce 3 intervals, as shown in Figure 1 .

In general, the overlapped sections for adjacent trajectories are not exactly coincident with each other, i.e., the start and end points of interval 2 in Figure 1 are not coincident for the two trajectories. In order to depict the adjacent trajectories with a continuous curve, a weighted synthesis method [36] is introduced. The effects of the curve trajectories $Q_{i}\left(t_{i}\right)$ and $Q_{i+1}\left(t_{i+1}\right)$ can be separated by respective weight functions $g(u)$, $h(u)$, and the equation can be revised to:

$$
\begin{aligned}
& P_{i+1}(t)=g(u) \cdot Q_{i}\left(t_{i}\right)+h(u) \cdot Q_{i+1}\left(t_{i+1}\right), \\
& g(u)=u, \quad h(u)=1-u \quad(0 \leq u \leq 1),
\end{aligned}
$$

where $P_{i+1}(t)$ denotes the weighted synthesis quadratic spline curve; $u$ is the parametric variable for the weight functions $g(u)$ and $h(u)$, ranging between 0 and $1 ; t_{i}$ and $t_{i+1}$ are the parametric variables for the quadratic spline curves $Q_{i}\left(t_{i}\right)$ and $Q_{i+1}\left(t_{i+1}\right)$, and they fall between 0 and 1 . In order to unify these parametric variables, replace $u, t_{i}$, and $t_{i+1}$ with parametric variable $t$ :

$$
\begin{aligned}
& t_{i}=t+0.5 \\
& t_{i+1}=t \quad(0 \leq t \leq 0.5) \\
& u=2 t .
\end{aligned}
$$

By solving the simultaneous equations, the mathematical expression can be summarized as follows:

$$
\begin{aligned}
P_{i+1}(t)= & \left(-4 t^{3}+4 t^{2}-t\right) P_{i}+\left(13 t^{3}-10 t^{2}+1\right) P_{i+1} \\
& +\left(-12 t^{3}+8 t^{2}+t\right) P_{i+2}+\left(4 t^{3}-2 t^{2}\right) P_{i+3} \\
i= & 1,2, \ldots, n-3 \quad(0 \leq t \leq 0.5) .
\end{aligned}
$$


An open quadratic spline curve with $n$ characteristic points consists of $n-3$ segments. Therefore, two endpoint constraints need to be added. The corresponding endpoint constraints are:

$$
P_{0}=P_{1}, \quad P_{n+1}=P_{n} .
$$

\subsection{Newton-cotes numerical integration}

Newton-Cotes numerical integration is employed to calculate the length of the curve, namely the distance between adjacent vehicles. It should be noted that the integral interval of $f(t)$ is within a finite interval $a \leq t \leq b$. The integral rules are defined by:

$$
\begin{aligned}
& L=\int_{a}^{b} f(t) d t \approx(b-a) \sum_{i=0}^{n} C_{i}^{(n)} f\left(t_{i}\right), \\
& f\left(t_{i}\right)=\left\|P_{i+1}\left(t_{i}\right)\right\|=\sqrt{\left[\phi^{\prime}\left(t_{i}\right)\right]^{2}+\left[\psi^{\prime}\left(t_{i}\right)\right]^{2}},
\end{aligned}
$$

where $L$ represents the space headway value and $n$ is the number of subintervals. Suppose that the integral interval $[a, b]$ is subdivided into $n$ subintervals $\left[t_{i}, t_{i+1}\right]$, of which $h=(b-a) / n$ by using the equally spaced nodes $t_{i}=a+i h$, for $i=0,1,2, \ldots, n . \quad C_{i}^{(n)}$ is the Cotes coefficient, which is defined through simple transformation of $s=(t-a) / h . C_{i}^{(n)}$ is independent of integrand $f(t)$ and integral interval $[a, b]$. It can be expressed as follows:

$$
\begin{aligned}
C_{i}^{(n)} & =\frac{1}{b-a} \int_{a}^{b} l_{i}(t) d t=\frac{h}{b-a} \int_{0}^{n} \prod_{\substack{j=0 \\
j \neq i}}^{n} \frac{s-j}{i-j} d s \\
& =\frac{(-1)^{n-i}}{i !(n-i) !} \frac{1}{n} \int_{0}^{n} \prod_{\substack{j=0 \\
j \neq i}}^{n}(s-j) d s .
\end{aligned}
$$

After following the procedure described above, space headway at turn movement trajectories will be estimated. The flow diagram of the computational process is briefly summarized in Figure 2.

\section{Case study}

In order to evaluate the accuracy of the proposed method, this study used data, which were supported by Next Generation Simulation (NGSIM), collected from Lankershim Boulevard in Universal City, a neighborhood of Los Angeles. The vehicles' trajectory data were transcribed from the video data at intersections using a software application. It detects and tracks vehicles from video images and transcribes the trajectory data to a database. As for the intersection, it generates many more traffic crashes than other locations due

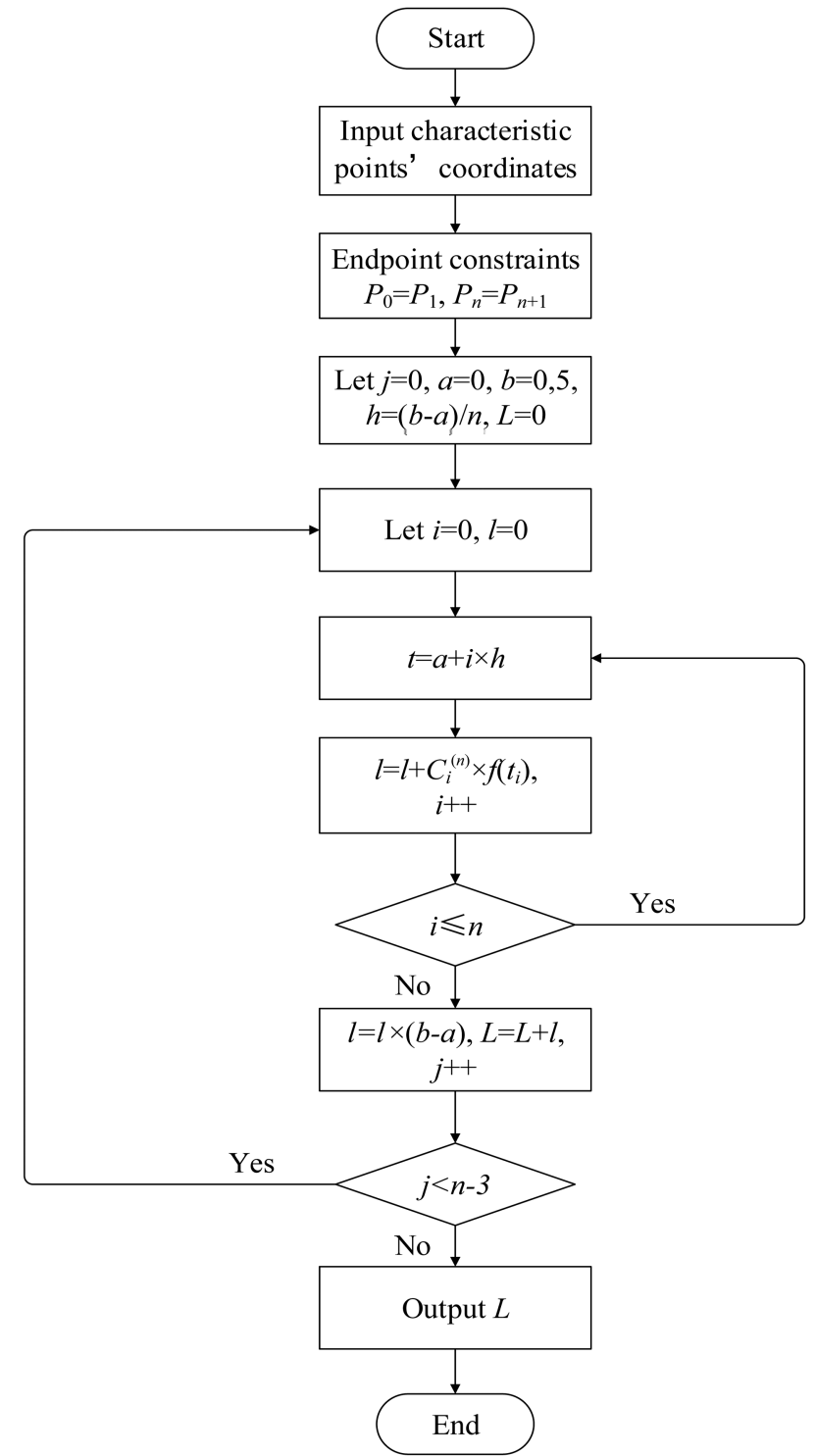

Figure 2. Flow diagram of the computational process.

to significant conflicts between motorized and nonmotorized traffics and pedestrians. In light of this, video cameras are commonly used at intersections to record traffic data and monitor traffic conditions. Thus, it is convenient to obtain vehicles' trajectory data using video cameras for this study.

The position and velocity of the vehicles in the NGSIM datasets had some noise. Thiemann et al. [37] reported such variations for all NGSIM datasets. To overcome this variation, positions and velocities were smoothed in each $0.5 \mathrm{~s}, 1 \mathrm{~s}$, and $4 \mathrm{~s}$ time period, respectively, using a moving average method in the following data analyses. Fifteen minutes (8:30 a.m. to $8: 45$ a.m.) of data including lateral $x$ coordinate, longitudinal $y$ coordinate, vehicle velocity and moving direction of the vehicle were selected every one-tenth of a second on June 16, 2005. Each vehicle also had information on the leading and following vehicles. 
Table 1. Traffic volume at the selected intersections.

\begin{tabular}{|c|c|c|c|c|c|c|c|c|c|c|c|c|}
\hline \multirow[t]{2}{*}{ Time period } & \multicolumn{3}{|c|}{ SB } & \multicolumn{3}{|c|}{ NB } & \multicolumn{3}{|c|}{ WB } & \multicolumn{3}{|c|}{ EB } \\
\hline & $\mathbf{L T}$ & TH & RT & LT & TH & RT & $\mathbf{L T}$ & TH & RT & LT & TH & RT \\
\hline 8:30-8:35 a.m. & 52 & 101 & 6 & 7 & 75 & 22 & 1 & 3 & 11 & 7 & 10 & 2 \\
\hline 8:35-8:40 a.m. & 41 & 108 & 13 & 4 & 120 & 28 & 0 & 2 & 17 & 6 & 10 & 0 \\
\hline $8: 40-8: 45$ a.m. & 46 & 115 & 9 & 4 & 88 & 30 & 4 & 3 & 11 & 12 & 14 & 1 \\
\hline Sum & 139 & 324 & 28 & 15 & 283 & 80 & 5 & 8 & 39 & 25 & 34 & 3 \\
\hline Percentage (\%) & 28.3 & 66.0 & 5.7 & 4.0 & 74.9 & 21.1 & 9.6 & 15.4 & 75.0 & 40.3 & 54.8 & 4.9 \\
\hline
\end{tabular}

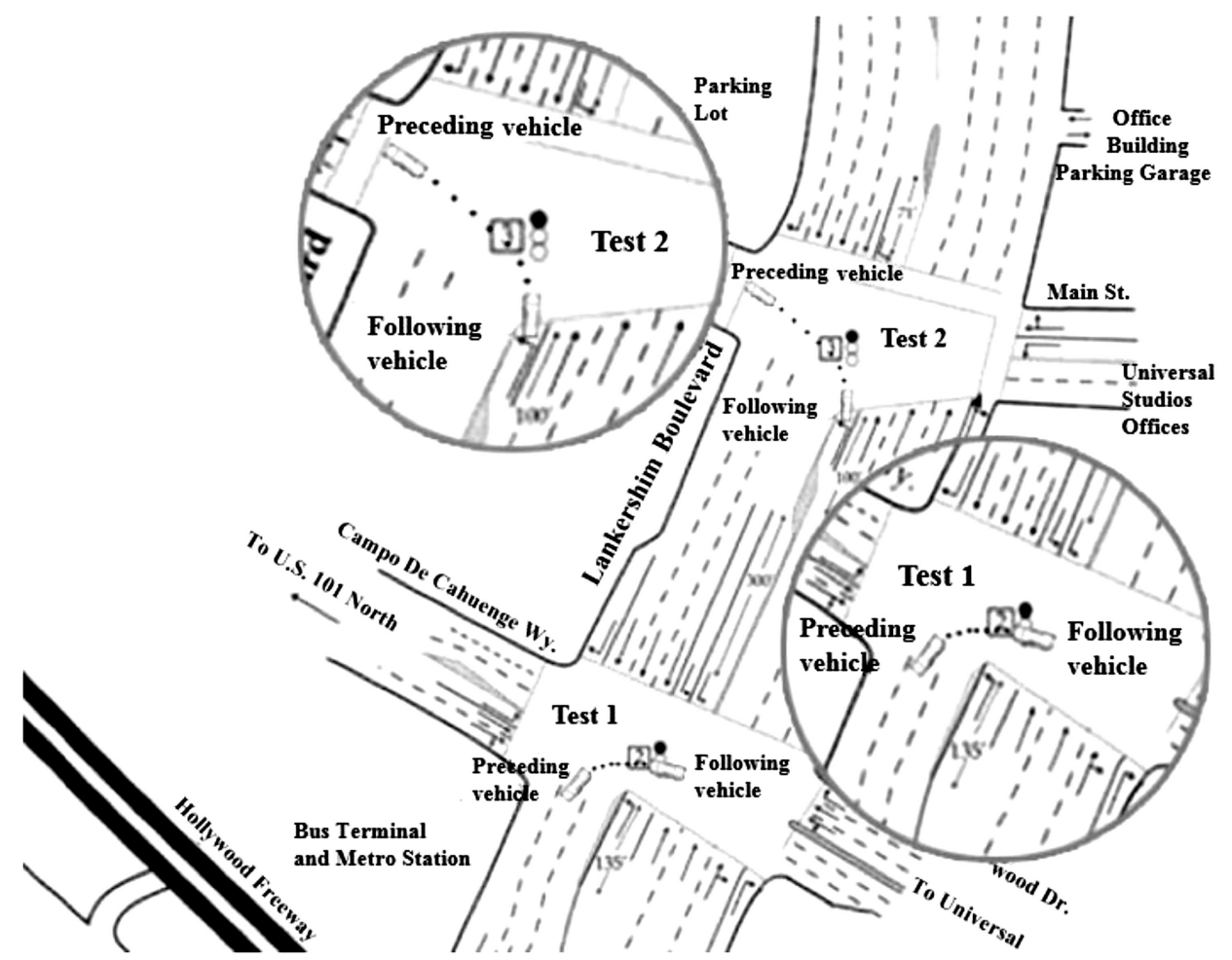

Figure 3. Schematic drawing of vehicle trajectories and characteristic points.

During the 15-minute observation period, there were 983 vehicles that passed through the intersections, among which 184 vehicles turned left $(18.7 \%)$ and 150 vehicles turned right (15.3\%) (Table 1).

As shown in Figure 3, the study area consists of two signalized intersections and three to four lane arterial segments. In this study, 120 turn movement trajectories (60 for left turns and 60 for right turns) were selected for performance evaluation. For each vehicle (sample), one trajectory was chosen at a random time when the turning vehicle was following a leading one in the intersection. One sample, providing the corresponding space headway value, represents one snapshot of the leader-follower position during their turning movement and that snapshot is taken at one random position among numerous continuous positions. In addition, two tests (i.e., two trajectories) were selected to provide visual validation and further examine the performance of the proposed method.

\subsection{Validation of model coefficients}

The following two steps are taken for discussing the appropriate number of characteristic points $N$ and Cotes coefficient $C_{i}^{(n)}$. In general, the number of characteristic points has a noteworthy impact on the results of the method's performance. Figure 4 depicts that the influence on the method's performance is dramatic and the Mean Absolute Percentage Error (MAPE) (as will be described in the following section) is unsatisfactory when $N$ is less than 8 . In contrast, when $N$ is greater than (or equal to) 9, the MAPE is not sensitive to the number of characteristic points and the error can be reduced significantly. However, in theory, complicated computations may be generated as the value of $N$ increases. In this study, $N=9$.

$C_{i}^{(n)}$ is a coefficient of the integral rules, which must be predetermined. As shown in Figure 5, MAPE varied greatly when $n$ is less than 4 . When $n$ is 


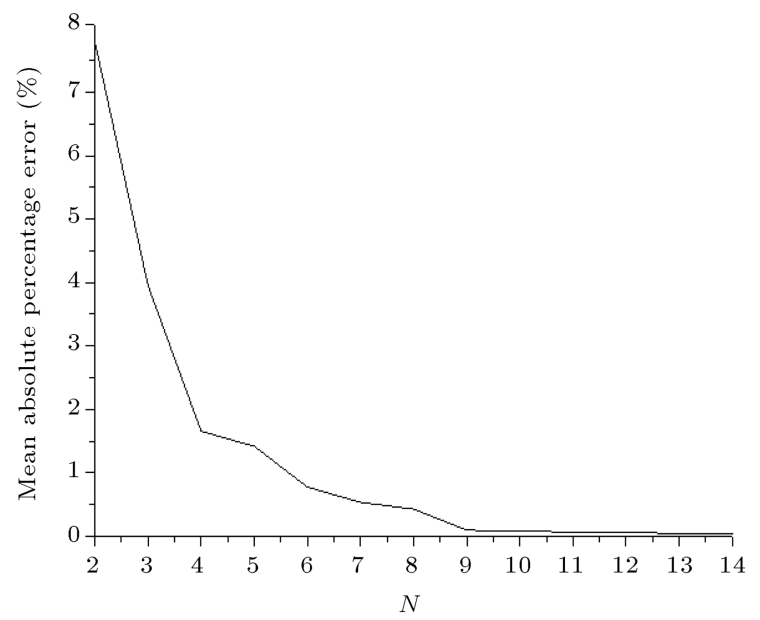

Figure 4. Impact of the number of characteristic points on method's performance.

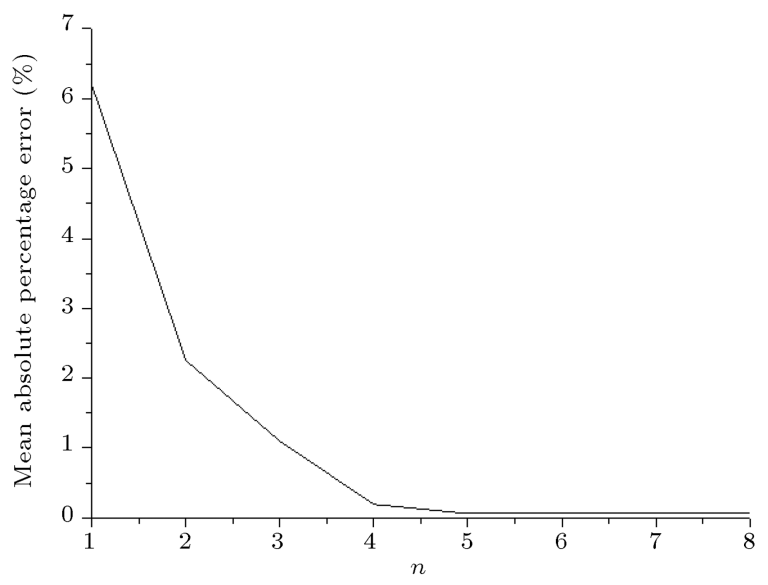

Figure 5. Impact of Cotes coefficient on method's performance.

greater than 4, the MAPE tends to be stable and is less than $0.1 \%$ for $n=5$, which is an acceptable error for such applications. When $n$ increases from 5 to
8, the MAPE only improves $0.009 \%$. Thus, $[5,+\infty]$ is a reasonable range for $n$. However, the NewtonCotes rule sometimes will suffer from catastrophic Runge's phenomenon [38], and the MAPE increases exponentially for large values of $n$. In order to avoid such a phenomenon and reduce computational load, $n$ is set to 5 in this case.

\subsection{Selection of characteristic points}

Based on the information collected for each $x$ coordinate, $y$ coordinate and moving direction, complete vehicle trajectories are transcribed at a resolution of 10 frames per second. Each frame provides a data point representing the location of the preceding vehicle. As mentioned above, nine characteristic points will be selected from those data points. When the preceding vehicle arrived at the last characteristic point, the following vehicle arrived at the first characteristic point. The remaining seven characteristic points, which were the trajectory coordinates of the preceding vehicle, were chosen to be as equally spaced as possible. The coordinates of the characteristic points for the two tests are displayed in Table 2.

\subsection{Comparison of results}

In addition to the proposed method, another algorithm based on computer vision and a trilinear method was selected for space headway calculation and comparison. The compared method has flexibility and adaptability characteristics. Without iteration and optimization calculations, an analytic expression of the external parameters can be established through mathematical derivation according to a pin-hole imaging model. As shown in Figure 6a, the comparison method is capable of acquiring accurate and reliable results in straight segments. However, in Figure 6b, it is quite difficult to accurately obtain the space headway at turn movement trajectories, mainly owing to the limitation of recti-

Table 2. Coordinates of the characteristic points.

\begin{tabular}{ccccc}
\hline & \multicolumn{2}{c}{ Test $\mathbf{1}(\mathbf{m})$} & \multicolumn{2}{c}{ Test $\mathbf{2}(\mathbf{m})$} \\
\cline { 2 - 5 } No. & $\boldsymbol{x}$ coordinate & $\boldsymbol{y}$ coordinate & $\boldsymbol{x}$ coordinate & $\boldsymbol{y}$ coordinate \\
\hline 1 & 4.623 & 131.892 & -15.462 & 337.470 \\
2 & 5.805 & 131.360 & -15.624 & 335.955 \\
3 & 7.138 & 130.777 & -16.198 & 334.332 \\
4 & 8.570 & 130.097 & -16.507 & 331.571 \\
5 & 9.284 & 129.615 & -17.341 & 328.585 \\
6 & 10.668 & 128.341 & -18.010 & 326.276 \\
7 & 11.328 & 127.540 & -20.138 & 324.529 \\
8 & 11.984 & 126.757 & -21.623 & 323.201 \\
9 & 13.386 & 125.068 & -22.895 & 322.408 \\
\hline
\end{tabular}


Table 3. Comparison of Mean Absolute Percentage Error (MAPE) for the 120 samples.

\begin{tabular}{|c|c|c|c|c|c|c|}
\hline \multirow[b]{2}{*}{ Direction } & \multicolumn{2}{|c|}{ LT (\%) } & \multicolumn{2}{|c|}{ RT (\%) } & \multicolumn{2}{|c|}{ Sum (\%) } \\
\hline & $\begin{array}{c}\text { Proposed } \\
\text { method }\end{array}$ & $\begin{array}{c}\text { Compared } \\
\text { method }\end{array}$ & $\begin{array}{c}\text { Proposed } \\
\text { method }\end{array}$ & $\begin{array}{c}\text { Compared } \\
\text { method }\end{array}$ & $\begin{array}{c}\text { Proposed } \\
\text { method }\end{array}$ & $\begin{array}{c}\text { Compared } \\
\text { method }\end{array}$ \\
\hline NB & 0.140 & 8.455 & 0.084 & 8.253 & 0.089 & 8.271 \\
\hline SB & 0.117 & 9.231 & 0.050 & 8.253 & 0.106 & 9.075 \\
\hline WB & 0.068 & 9.060 & 0.067 & 8.618 & 0.068 & 8.874 \\
\hline EB & 0.102 & 8.735 & 0.083 & 8.442 & 0.096 & 8.655 \\
\hline Total & 0.107 & 9.082 & 0.078 & 8.311 & 0.093 & 8.697 \\
\hline
\end{tabular}

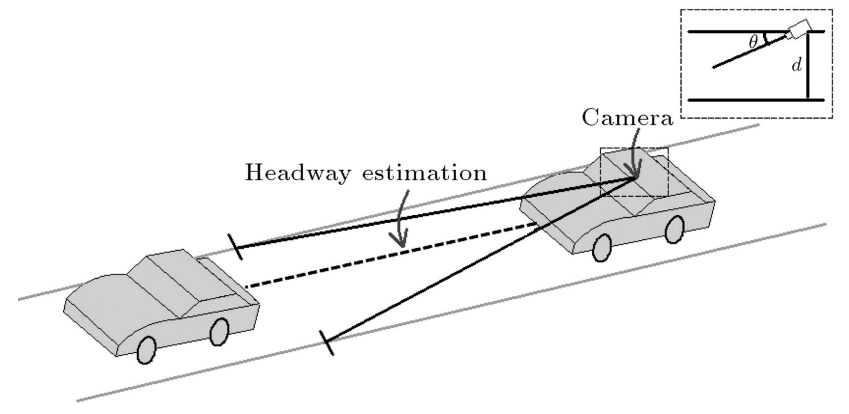

Figure 6a. Headway estimation in straight segment of roadway.

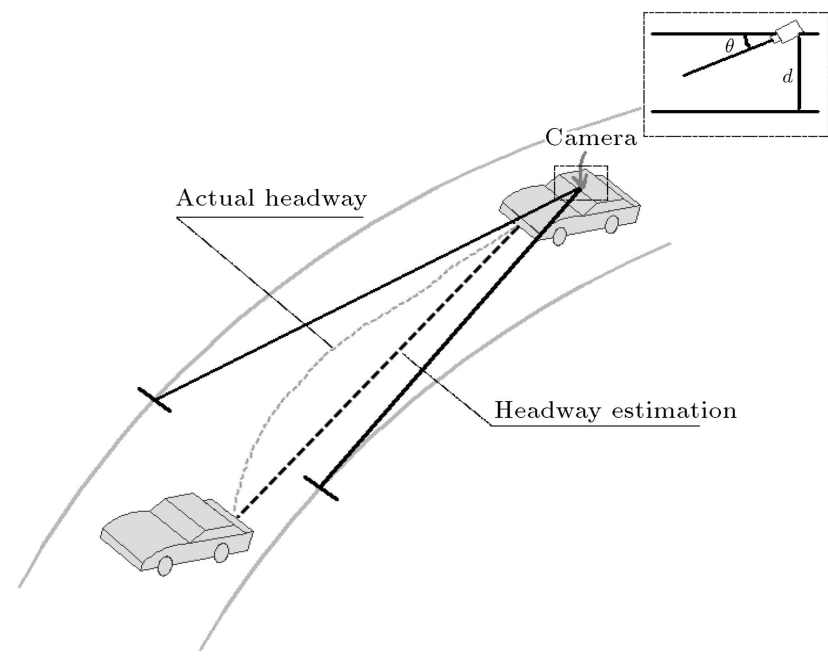

Figure 6b. Headway estimation at turn movement trajectories.

linear propagation. Several Measures Of Effectiveness (MOEs) including MAPE and Error Metric (EM) were chosen to evaluate the performance of the two methods. To avoid overrating the discrepancies for large distance, the EM was weighted by logarithm and squared. These measures are calculated as follows:

$$
\begin{aligned}
& M A P E=\frac{1}{M} \sum_{k=1}^{M}\left|\frac{\hat{L}_{k}-L_{k}}{L_{k}}\right|, \\
& E M=\sqrt{\sum_{k=1}^{M}\left(\log \frac{\hat{L}_{k}}{L_{k}}\right)^{2}},
\end{aligned}
$$

where $M$ denotes the sample size and $L_{k}$ and $\hat{L_{k}}$ are actual and estimated space headways, respectively.

Table 3 shows comparative results of the proposed and compared methods. The 120 samples consist of four left turns and 42 right turns (NB), 43 left turns and seven right turns (SB), five left turns and eight right turns (WB), and eight left turns and three right turns (EB). The results show that the proposed method produces significant improvements over the compared method for each direction. In total, the proposed method has an MAPE of $0.093 \%$ with an $8.604 \%$ improvement from the compared method. In addition, for the proposed method, the results of right-turning vehicles are better than those of left-turning vehicles. These results can be attributed to the fact that rightturning trajectories have simpler linetype than those of left-turning vehicles.

In order to better evaluate the performance of this method, the researchers selected two example paths among all test samples for detailed testing and analysis. Table 4 presents the MOEs for two example paths. According to Test 1, it is the proposed method that has more accurate and reliable results (with $0.083 \%$ of MAPE) than the compared method (with $8.330 \%$ of MAPE). Likewise, the MAPE of Test 2 by the proposed method and the compared method produce different performance, i.e., $0.082 \%$ and $7.781 \%$, respectively. With regard to the average, the proposed method also has excellent performance with 0.004 of EM. However, the performance of the competing method is unsatisfactory, mainly owing to the limitation of rectilinear propagation. The space headway at turn movement trajectories is fitted by a straight line, which is difficult to use to indicate the general shape of the trajectories, although the trilinear method is capable of acquiring accurate and reliable results in straight segments of roadway. Hence, the trilinear method is inferior at turn movement trajectories.

In order to provide a visual validation of this method's performance, vehicle path estimation results of two example paths are presented in Figure 7, showing the real trajectories and those from the proposed and compared methods. The vehicle path of the proposed method is specified by a sequence of quadratic spline curve segments. As shown in Figure 7(a) and (b), the 
Table 4. Comparison of Measures of Effectiveness (MOEs).

\begin{tabular}{|c|c|c|c|c|c|c|c|}
\hline \multirow{3}{*}{ Method } & \multicolumn{3}{|c|}{ Test $1(\mathbf{m})$} & \multicolumn{3}{|c|}{ Test $2(\mathbf{m})$} & \multirow{3}{*}{$\frac{\text { Average }}{\text { EM }}$} \\
\hline & \multicolumn{2}{|c|}{ Space headway value } & \multirow{2}{*}{$\begin{array}{c}\text { MAPE } \\
(\%)\end{array}$} & \multicolumn{2}{|c|}{ Space headway value } & \multirow{2}{*}{$\begin{array}{c}\text { MAPE } \\
(\%)\end{array}$} & \\
\hline & Estimated & Actual & & Estimated & Actual & & \\
\hline Proposed method & 11.790 & 11.800 & 0.083 & 17.786 & 17.801 & 0.082 & 0.004 \\
\hline Compared method & 10.817 & 11.800 & 8.330 & 16.416 & 17.801 & 7.781 & 0.048 \\
\hline
\end{tabular}

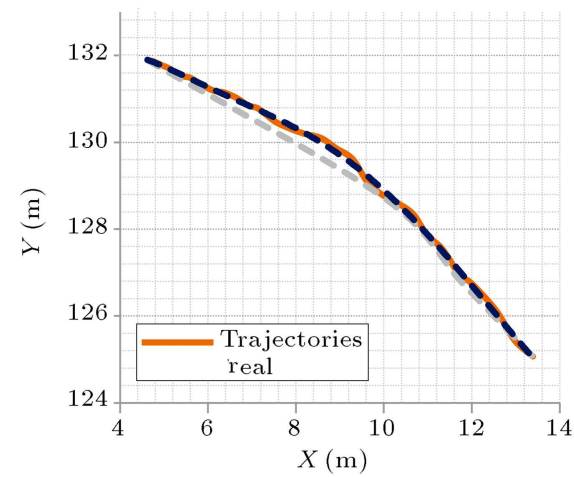

(a) Test 1

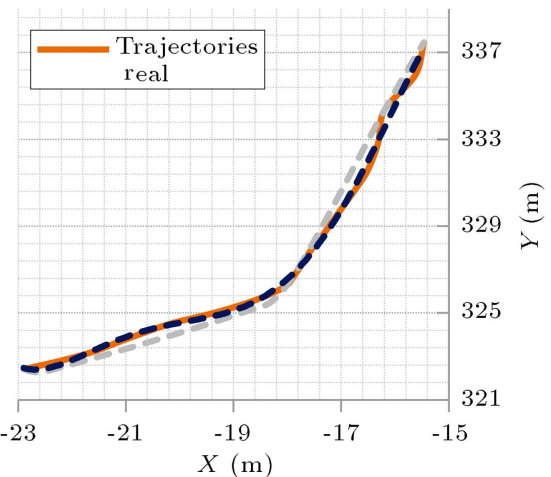

(b) Test 2

Figure 7. Schematic drawing of actual vehicle path and estimated vehicle path.

trajectory of the proposed method and the real curve are almost identical, confirming the high accuracy of the model. The performance of the competing method, however, is inferior, indicating that it is quite difficult to accurately depict the space headway at turn movement trajectories.

\subsection{Analysis and discussion}

The proposed method was also applied to investigate the effects of vehicle speed, relative speed of vehicles, and time period on the spacing of vehicles in carfollowing. For car-following, space headway is different from space gap. However, the distinction is often ignored even though it is relevant. The space headway is the distance between the front bumpers of two consecutive vehicles. In contrast, space gap denotes the rear-bumper-to-front-bumper distance which is relevant for car-following [39]. To avoid confusion in car-following, the space gap (not space headway) will be analyzed and discussed in this section. It can be obtained easily by the following equation:

space gap $=$ space headway

-length of the leading vehicle

Table 5 provides the average gap of vehicles in three time slots including 8:30-8:35 a.m., 8:35-8:40 a.m., and 8:40-8:45 a.m. Space and time gaps are also analyzed based on different vehicle speeds (i.e.,

Table 5. Space and time gap at different speeds.

\begin{tabular}{|c|c|c|c|c|c|}
\hline \multirow[t]{2}{*}{ Gap } & \multirow[t]{2}{*}{ Time period } & \multicolumn{3}{|c|}{ Speed } & \multirow[t]{2}{*}{ Average gap } \\
\hline & & $<8 \mathrm{~km} / \mathrm{h}$ & $8-32 \mathrm{~km} / \mathrm{h}$ & $>32 \mathrm{~km} / \mathrm{h}$ & \\
\hline \multirow{4}{*}{$\begin{array}{l}\text { Space gap } \\
(\mathrm{m})\end{array}$} & $8: 30-8: 35$ a.m. & 5.22 & 24.97 & 39.58 & 24.85 \\
\hline & $8: 35-8: 40$ a.m. & 5.32 & 26.50 & 42.18 & 28.06 \\
\hline & $8: 40-8: 45$ a.m. & 6.87 & 27.04 & 40.52 & 28.73 \\
\hline & Average & 5.63 & 25.25 & 41.08 & 26.79 \\
\hline \multirow{4}{*}{$\begin{array}{c}\text { Time gap } \\
(\mathrm{s})\end{array}$} & $8: 30-8: 35$ a.m. & 15.20 & 5.05 & 3.15 & 6.71 \\
\hline & $8: 35-8: 40$ a.m. & 20.93 & 5.38 & 3.30 & 7.69 \\
\hline & $8: 40-8: 45$ a.m. & 20.36 & 5.22 & 3.21 & 6.82 \\
\hline & Average & 18.43 & 4.95 & 3.22 & 7.31 \\
\hline
\end{tabular}


less than $8 \mathrm{~km} / \mathrm{h}(5 \mathrm{mph}), 8$ to $32 \mathrm{~km} / \mathrm{h}$ (5 to 20 $\mathrm{mph})$, and more than $32 \mathrm{~km} / \mathrm{h}(20 \mathrm{mph}))$. The analysis showed the impacts of the subject vehicle speed on its gap in each car-following combination. The space gap increased and time gap decreased as the vehicle speed increased.

Car-following is a control process that a driver of a following vehicle uses to maintain a safe distance behind the vehicle ahead by using either acceleration or deceleration in response to the actions of the leading vehicle. In essence, drift and goal-seeking behavior of a pair of vehicles is related to how the space gap between the leading and following vehicles oscillates around what might be termed as stable space gap [40]. This behavior happens because of the constraints of vehicle dynamics and human maneuvers. For example, the driver of the following vehicle may not be able to judge the leading vehicle's speed accurately or maintain its own speed precisely. The drift and goal-seeking behavior can be illustrated by plotting relative spacing against relative speed, as shown in Figure 8 . The lateral coordinate represents the relative speed of the vehicles, and the longitudinal coordinate shows the distance to the preceding vehicle. The data points appearing in the negative regions correspond to the following vehicle traveling at speeds greater than the leading vehicle.

To discover the process of car-following at an intersection, Figures $9 \mathrm{a}$ and $9 \mathrm{~b}$ depict the driving behavior between the leading and following vehicles. The speed range during the process was between 0 and $58 \mathrm{~km} / \mathrm{h}$. The vehicle came to a complete stop between $22.3 \mathrm{~s}$ and $54.2 \mathrm{~s}$, indicating that the vehicle had a traffic signal delay of about $32 \mathrm{~s}$ at the intersection. The relative distance to the leading vehicle is presented in Figure 9a. The figure shows that the initial distance to the leading vehicle was $23.8 \mathrm{~m}$. The following vehicle was able to drive at a free-flow speed until approximately $14 \mathrm{~s}$ into the process when the space gap

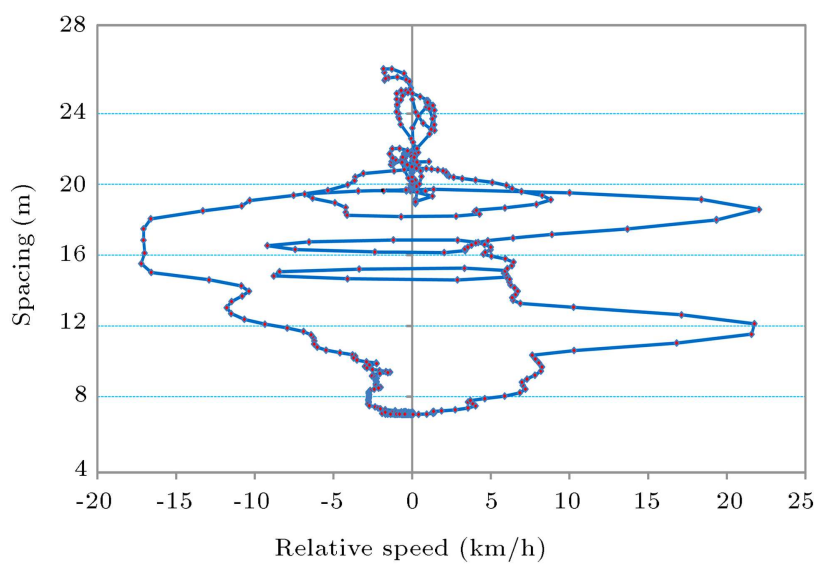

Figure 8. Car-following phase-space diagram using the proposed method.

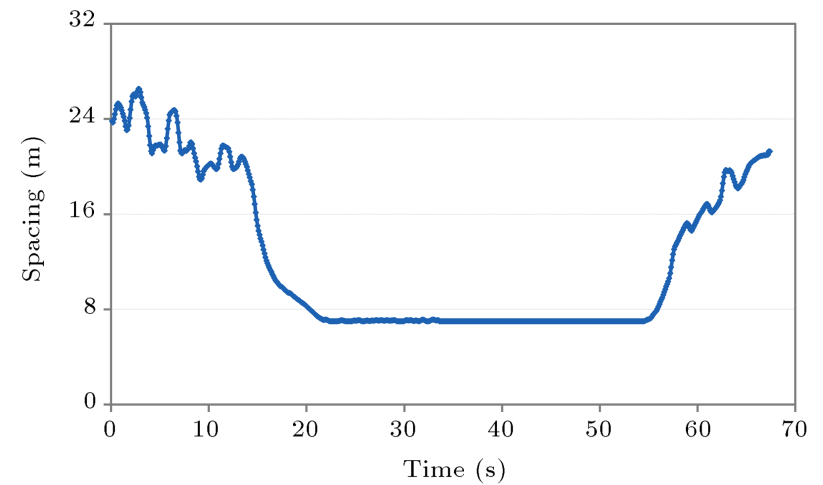

Figure 9a. Profile of space gap behind leading vehicle during car-following.

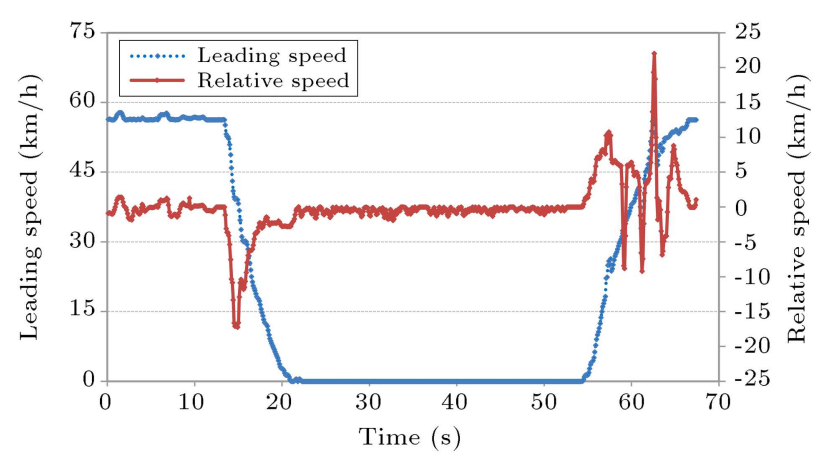

Figure 9b. Speed profile of leading and following vehicles during car-following.

diminished dramatically. In addition, Figure 9(b) also shows that the relative speed became unstable when the vehicle began to stop or accelerate away from the intersection.

\section{Conclusions}

This study proposed a hybrid model based on spline curves and numerical integration to calculate the space headway at turn movement trajectories. It had the advantages of simplifying the operation process and achieving more reliable and accurate results. The method was established by using data from vehicle trajectories, which were collected from videos at intersections. The space headway at turn movements was analogous to the track of the vehicle, which could be fitted by a quadratic spline curve. In addition, Newton-Cotes numerical integration was employed to calculate the distance, which was sufficient to address the question due to its meshing flexibility and ease of implementation.

In order to rigorously evaluate the accuracy of the proposed method and compare it with the trilinear method based on computer vision technology, this study used data collected from Lankershim Boulevard in the Universal City neighborhood of Los Angeles. The proposed method outperformed the competing 
method in calculating distance to the leading vehicle. In this study, 120 turn movement trajectories (60 for left turns and 60 for right turns) were chosen for performance evaluation. The proposed method produced an almost perfect estimated result, which decreased NB by $8.182 \%$ MAPE, SB by $8.969 \%$ MAPE, WB by $8.806 \%$ MAPE, EB by $8.559 \%$ MAPE, and total by $8.604 \%$ MAPE compared with the trilinear method. In addition, two tests were selected to further evaluate the performance of this method. The proposed method provided $0.083 \%$ MAPE for Test 1 , $0.082 \%$ MAPE for Test 2, and 0.004 Error Metric (EM) on average. The EM value was significantly lower than that from the competing method (with 0.048 of EM). To provide visual validation of the proposed method, vehicle path estimation results for two example paths were shown. These results confirmed the high accuracy of the hybrid model. The proposed method was applied to investigate the effects of vehicle speed, relative speed of vehicles, and time period on the spacing of vehicles during car-following. In addition, the proposed algorithm performed differently with different number of characteristic points $N$ and Cotes coefficient $C_{i}^{(n)}$, which illustrated the importance of defining parameters. In this case, $N=9, n$ was set to 5 .

The robustness of the method needs to be further verified through the utilization of different vehicle trajectories in different conditions such as skewed intersections. Different conditions may have impacts on the performance of the proposed method. Therefore, further research is necessary to investigate their impacts.

\section{Acknowledgement}

This study was sponsored by the Scientific Research Foundation of the Graduate School of Southeast University (No. YBJJ1633), the Fundamental Research Funds for the Central Universities, the Scientific Research Program of the Graduate School of Jiangsu Province (No. KYLX16-0280), and China Scholarship Council (CSC).

\section{References}

1. Al-Kaisy, A. and Durbin, C. "Platooning on two-lane two-way highways: an empirical investigation", J. Adv. Transport., 43(1), pp. 71-88 (2009).

2. Zhao, L. and Chien, S.I. "Investigating the impact of stochastic vehicle arrivals to optimal stop spacing and headway for a feeder bus route", J. Adv. Transport., 49, pp. 341-357 (2015).

3. Behnood, A., Modiri-Gharehveran, M., and Roshandeh, A.M. "The effects of drivers' behavior on driverinjury severities in Iran: An application of the mixedlogit model", Sci. Iran., 23(6), pp. 2429-2440 (2016).
4. Steux, B., Laurgeau, C., Salesse, L., et al. "Fade: A vehicle detection and tracking system featuring monocular color vision and radar data fusion", Proc. IEEE Intell. Vehicles Symp., 2, pp. 632-639 (2002).

5. Soudbakhsh, D., Eskandarian, A., and Chichka, D. "Vehicle collision avoidance maneuvers with limited lateral acceleration using optimal trajectory control", J. Dyn. Syst. Meas. Contr., 135(4), p. 041006 (2013).

6. Toledo-Moreo, R. and Zamora-Izquierdo, M.A. "Collision avoidance support in roads with lateral and longitudinal maneuver prediction by fusing GPS/IMU and digital maps", Transport. Res. C-Emerg. Technol., 18(4), pp. 611-625 (2010).

7. Wang, J., Zhang, L., Zhang, D., et al. "An adaptive longitudinal driving assistance system based on driver characteristics", IEEE Trans. Intell. Transp. Syst., 14(1), pp. 1-12 (2013).

8. McCall, J.C. and Trivedi, M.M. "Video-based lane estimation and tracking for driver assistance: survey, system, and evaluation", IEEE Trans. Intell. Transp. Syst., 7(1), pp. 20-37 (2006).

9. Huang, A. and Teller, S. "Probabilistic lane estimation for autonomous driving using basis curves", Auton. Robots, 31, pp. 269-283 (2011).

10. Gunay, B. "Using automatic number plate recognition technology to observe drivers' headway preferences", J. Adv. Transport., 46, pp. 305-317 (2012).

11. Aghabayk, K., Sarvi, M., Forouzideh, N., et al. "Modelling heavy vehicle car-following behavior in congested traffic conditions", J. Adv. Transport., 48, pp. 10171029 (2014).

12. Li, K. and Gao, Z. "An improved car-following model for railway traffic", J. Adv. Transport., 47, pp. 475-482 (2013).

13. Sarvi, M. "Heavy commercial vehicles-following behavior and interactions with different vehicle classes", $J$. Adv. Transport., 47, pp. 572-580 (2013).

14. Beliveau, Y.J., Fithian, J.E., and Deisenroth, M.P. "Autonomous vehicle navigation with real-time 3D laser based positioning for construction", Autom. Construct., 5(4), pp. 261-272 (1996).

15. Streller, D., Furstenberg, K., and Dietmayer, K. "Vehicle and object models for robust tracking in traffic scenes using laser range images", Proc. IEEE 5th Int. Conf. Intell. Transport. Syst., pp. 118-123, Singapore (2002).

16. Carullo, A. and Parvis, M. "An ultrasonic sensor for distance measurement in automotive applications", IEEE Sensors J., 1(2), pp. 143-147 (2001).

17. Clark, S. and Durrant-Whyte, H. "Autonomous land vehicle navigaton using millimeter wave radar", Int. Conf. Robot. Automat., 4, pp. 3697-3702 (1998).

18. Truong, Q.B. and Lee, B.R. "New lane detection algorithm for autonomous vehicles using computer vision", Proc. Int. Conf. Control Autom. Syst., pp. 1208-1213 (2008). 
19. Stauffer, C. and Grimson, W.E.L. "Adaptive background mixture models for real-time tracking", Proc. Computer Vision and Pattern Recognition, 2, pp. 246252 (1999).

20. Kilger, M. "A shadow handle in a video-based real-time traffic monitoring system", Proc. IEEE Workshop on Applications of Computer Vision, pp. 11-18 (1992).

21. Zhao, H., Liu, Z., and Zhang, H. "Segmentation of multiple moving targets based on modified C-V model", J. Sci. Instrum., 31(5), pp. 1082-1089 (2010).

22. Galic, S. and Loncaric, S. "Spatio-temporal image segmentation using optical flow and clustering algorithm", First International Workshop on Image and Signal Processing and Analysis, pp. 63-68 (2000).

23. Masoud, O., Papanikolopoulos, N.P., and Kwon, E. "The use of computer vision in monitoring weaving sections", IEEE Trans. Intell. Transp. Syst., 2(1), pp. 18-25 (2001).

24. Wu, B., Chen, W., Chang, C., et al. "A new vehicle detection with distance estimation for lane change warning systems", Proc. IEEE Intell. Vehicles Symp., pp. 698-703 (2007).

25. Wang, W., Lu, M., Kao, H., et al. "Nighttime vehicle distance measuring systems", IEEE Trans. Circuits Syst. II, 54(1), pp. 81-85 (2007).

26. Chen, Y., Das, M., and Bajpai, D. "Vehicle tracking and distance estimation based on multiple image features", Proc. 4th Can. Conf. Comput. Robot Vis., pp. 371-378 (2007).

27. Zhang, Q. and Xie, M. "Study on the method of measuring the preceding vehicle distance based on trilinear method", Proc. 2nd Int. Conf. Comput. Modeling Simul., 2, pp. 475-479 (2010).

28. Wu, C., Lin, C., and Lee, C. "Applying a functional neurofuzzy network to real-time lane detection and front-vehicle distance measurement", IEEE Trans. Syst. Man Cybern. C Appl. Rev., 42(4), pp. 577-589 (2012).

29. Zhao, X., Zhang, C., Yang, B., et al. "Adaptive knot placement using a GMM-based continuous optimization algorithm in B-spline curve approximation", Comput.-Aided Des., 43(6), pp. 598-604 (2011).

30. Park, H. and Lee, J.H. "B-spline curve fitting based on adaptive curve refinement using dominant points", Comput-Aided Des., 39, pp. 439-451 (2007).

31. Kouibia, A. and Pasadas, M. "Approximation by smoothing variational vector splines for noisy data", J. Comput. Appl. Math., 211, pp. 213-222 (2008).

32. Jahanpour, J. "High speed contouring enhanced with C-2 PH quintic spline curves", Sci. Iran., 19(2), pp. 311-319 (2012).

33. Rostami, S., Shojaee, S., and Saffari, H. "An explicit time integration method for structural dynamics using cubic B-spline polynomial functions", Sci. Iran., 20(1), pp. 23-33 (2013).
34. Leader, J., Numerical Analysis and Scientific Computation, MA, Addison-Wesley (2004).

35. Hearn, D., Baker, M.P., and Carithers, W.R., Computer Graphics with Open GL, Fourth Edition, Pearson Education (2011).

36. Aczel, J. "On weighted synthesis of judgments", Aequationes Math., 27(1), pp. 288-307 (1984).

37. Thiemann, C., Treiber, M., and Kesting, A. "Estimating acceleration and lane-changing dynamics from next generation simulation trajectory data", Transp. Res. Rec., 2088, pp. 90-101 (2008).

38. Dahlquist, G. and Bjork, A. "Equidistant interpolation and the Runge phenomenon", Numer. Meth., 1, pp. 101-103 (1974).

39. Kesting, A., Traffic Flow Dynamics: Data, Models and Simulation, Springer (2013).

40. Chakroborty, P. and Kikuchi, S. "Evaluation of the general motors based car-following models and a proposed fuzzy inference model", Transport. Res. CEmerg. Technol., 7(4), pp. 209-235 (1999).

\section{Biographies}

Chao Wang received his $\mathrm{PhD}$ degree in Transportation Engineering from Southeast University in 2018. During 2017-2018, he went to Purdue University as a visiting $\mathrm{PhD}$. He is now a research Assistant Professor at School of Transportation, Southeast University. His research interests include public transport, intelligent transportation systems, and transport modeling \& optimization.

Zhirui Ye earned his $\mathrm{PhD}$ degree in Transportation Engineering from Texas A\&M University, College Station, Texas, 2007. He is now a Professor in the School of Transportation and Director of the Transportation Research Institute, Southeast University. His research interests include, but are not limited to, advanced public transportation, traffic safety, and connected vehicles.

Enhui Chen is now a $\mathrm{PhD}$ Candidate in Transportation Engineering at Southeast University. His research interests include traffic safety and intelligent transportation systems.

Jiaxiao Feng is now a $\mathrm{PhD}$ Candidate in Transportation Engineering at Southeast University. Her research interests include public transportation and transportation system modeling. 